\title{
Fabrication and electrorotation of novel epoxy based micromotor working in uniform DC electric field
}

\author{
Rita A. Bauer ${ }^{1}$, Lóránd Kelemen ${ }^{2}$, Masami Nakano ${ }^{3}$, Atsushi Totsuka ${ }^{3}$ and Miklós \\ Zrínyi ${ }^{1,4}$ \\ ${ }^{1}$ Department of Biophysics and Radiation Biology, Semmelweis University \\ H-1089 Budapest, Nagyvarad ter 4., Hungary \\ ${ }^{2}$ Biological Research Centre, Hungarian Academy of Sciences \\ H-6726 Szeged, Temesvári krt. 62., Hungary \\ ${ }^{3}$ Intelligent Fluid Control Systems Laboratory, Institute of Fluid Science, Tohoku University \\ 2-1-1 Katahira, Aoba-ku, Sendai, 980-8577, Japan \\ ${ }^{4}$ MTA-SE Molecular Biophysics Research Group, Department of Biophysics and Radiation \\ Biology, Semmelweis University, H-1089 Budapest, Nagyvarad ter 4., Hungary \\ E-mail of corresponding author: mikloszrinyi@gmail.com
}

\begin{abstract}
We have presented the first direct observation of electric field induced rotation of epoxy based polymer rotors. Polymer disks, hollow cylinders and gears were prepared in few micrometer dimensions as rotors. Electrorotation of these sub-millimeter sized tools was studied under uniform DC electric field. The effects of shape, size and thickness were investigated. The novel epoxy based micro devices show intensive spinning in uniform DC electric field. The rotational speed of micron-sized polymer rotors can be conveniently tuned in wide range (between $300-3000 \mathrm{rpm}$ ) by the electric field intensity, opening new perspectives for their use in several MEMS applications.
\end{abstract}




\section{Introduction}

The rapid development of micro-manufacturing has initiated the development of electrostatic micromotor to be used in micro-electromechanical systems (MEMS) for energy conversion. The early attempts to miniaturize the electromotors were achieved by making a scale reduction of existing motor parts. On the basis of this technique the first micromotor was developed by L.S Fan and Y.C. Tang in 1988 [1]. Their electrostatic driven motor had a diameter of $120 \mu \mathrm{m}$ and the rotation speed of $500 \mathrm{rpm}$ with a torque of some $\mathrm{pNm}$. Due to their complicated construction, the coils of traditional electromagnetic motor are rather difficult to miniaturize. Despite several works have been devoted to develop and manufacture micromotors, there are only few studies available in literature about their design and performance [1-5]. One of the constructions is the electrostatic side-drive motor which is driven by electrostatic forces that create mechanical torque. The rotary movement of this motor is due to attractive interactions between stator electrodes and teeth type of rotor electrodes [3].

In this work a novel principle has been proposed for realization of a side drive micromotor, based on the electrorotation phenomena. Electrorotation or Quincke rotation is the circular movement of an electrically polarized micron sized particle or material in dielectric liquids [6,7].

In 1896 G. Quincke observed that some solid particles can spontaneously rotate in certain media if a large uniform electric field is applied [6]. The rotational axis is perpendicular to the electrostatic field. The phenomenon exhibits a threshold value of the electric field and occurs only if certain conditions concerning the conductivity and permittivity of the particles and of the liquid are satisfied [7,8]. This condition for spontaneous rotation can be expressed by the permittivity, $\varepsilon$ and conductivity, $\sigma$ data: $\varepsilon_{2} \sigma_{1}<\varepsilon_{1} \sigma_{2}$ where the subscripts 1 and 2 refer to the liquid and the dispersed particle, respectively. Due to these special criteria of material characteristics, it is not easy to observe the phenomenon. Only a limited number of experimental and theoretical studies are available. F. Peters, L. Lobry, A. Khayari, and E. Lemaire have studied the electrorotation of spherical and infinite insulating cylinder [9]. They found for spherical particles that if the rotor size is much larger than the characteristic ion layer thickness around the particles the angular velocity does not depend on the rotor size. The angular velocity, $\omega$ as a function of the applied field, $E$ can be expressed as [7-9]:

$$
\omega(E)= \pm \frac{1}{\tau_{M W}} \sqrt{\frac{E^{2}}{E_{c r}^{2}}-1} \quad E>E_{c r}
$$

where $\tau_{\mathrm{MW}}$ is the Maxwell-Wagner interfacial polarization relaxation time for spheres defined as

$$
\tau_{M W}=\left(2 \varepsilon_{1}+\varepsilon_{2}\right) /\left(2 \sigma_{1}+\sigma_{2}\right)
$$

The \pm sign in Equation (1) takes into account the two possible directions of rotation. In the opposite case when 
the thickness of the cylindrical rotor may not be neglected, they have found size dependence [9]. They concluded that the size effect originates from ion diffusion and electro migration in the charged layer.

In our previous work, we have reported the development of micron-sized polymer composites disks that showed electrorotation in uniform DC and AC electric field [10-12].

The present paper deals with the first direct observation of electric field induced rotation of epoxy based polymer rotors with variable size and shape. Our main purpose was to fabricate rotors, which could be used as main building blocks of electro-micromotor. The rotor speed as a function of uniform DC field as well as the effect of shape and size of the micro rotors on the rotary motion has been studied.

\section{Materials and Method}

The SU8 2075 photoresists and SU8 developer (mr-Dev 600) were purchased from Micro Resist Technology (Germany). Microscope glass slides were purchased from Menzel Glaser (Germany).

The SU8 disks were made of SU8 2075 at two different thicknesses and four different diameters. First, the microscope cover slide substrates were cleaned in chromic acid for several days, then thoroughly rinsed in distilled water and finally dried in a stream of nitrogen. Immediately before their coating with SU8 the substrates were oxygen plasma treated for $10 \mathrm{~min}$ in $29.6 \mathrm{~W}$ field. The thickness of the SU8 was set by spreading $300 \mu \mathrm{L}$ of SU8 2075 onto the substrate with spin coating technique with rotational speeds of 3000 and $5000 \mathrm{rpm}$. The layers then were placed in dark on a $65^{\circ} \mathrm{C}$ hot plate for $120 \mathrm{~min}$ and onto a $95^{\circ} \mathrm{C}$ one for overnight.
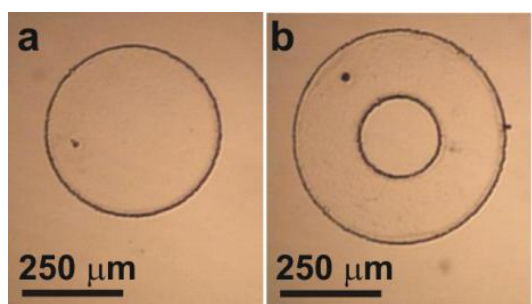

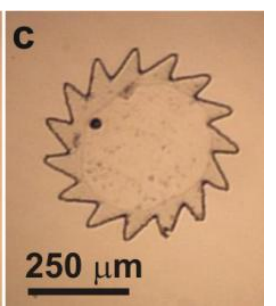

Figure 1: Polymer rotors prepared from epoxy based polymer. (a) polymer disk, (b) hollow cylinder, (c) gear with asymmetric teeth wheels. The black dot close to the edge of the micro tools was used to assist the determination of the speed of rotation

The next day the layers were illuminated by a flood exposure lamp filtered for the $365 \mathrm{~nm} \mathrm{Hg}$ line at $10000 \mathrm{~mJ} / \mathrm{cm}^{2}$ (model 97435, Newport, Irvine, Ca, USA) though a mask defining three different shapes: disk, ring (hollow cylinder), gear with asymmetric teeth (Figure 1). Their diameter was 100, 300, 400 and $500 \mu \mathrm{m}$. The illuminated sample was then heat-treated first at $65{ }^{\circ} \mathrm{C}$ for $30 \mathrm{~min}$ then at $95{ }^{\circ} \mathrm{C}$ for $60 \mathrm{~min}$. The development was carried out by immersing the substrate in clean SU8 developer 3 times, 5 min each. This was followed by washing in 1:1 ratio of ethanol- $\mathrm{H}_{2} \mathrm{O}$ mixture, drying and hard-baking at $95{ }^{\circ} \mathrm{C}$ for $30 \mathrm{~min}$. Then, the disks still on the substrate were marked by a short illumination with an ultrashort-pulsed laser to improve the observation of their rotation. The resulted hard SU8 rings were then removed mechanically from the substrate into acetone and finally transferred into a plastic container for storage and transportation. 
Figure 2 shows an experimental device to apply electric field to the polymer rotors. The gap distance between the electrodes was $3 \mathrm{~mm}$. The space between the electrodes was filled up with special oil which contains substantial amount of triglyceride of oleic-, palmitic-, and linoleic acids, with conductivity of $\sigma_{1}=9.56 \cdot 10^{-10} \mathrm{~S} / \mathrm{m}$ and relative permittivity of $\varepsilon_{1}=3.32$. The electric field was supplied by a high voltage DC power supply (TREC, USA). The intensity of the uniform DC electric field was increased step by step from $0.5 \mathrm{kV} / \mathrm{mm}$ up to $2.2 \mathrm{kV} / \mathrm{mm}$ and it was applied perpendicularly to the axis of the rotors. Although in our experiments high voltage was involved but it is really the high electric field which is responsible for the effect.

The rotation of floating micro-rotors in the oil was followed by optical microscope (OLYMPUS, Japan) equipped by a high speed camera (4500 fps, Photron, Japan). In order to visualize the rotation and to determine the angular frequency, a visible sign close to the edge of the disk was used (see Figure 1). The angular frequency of the rotation was determined by recording the spinning motion of the disk on the high speed camera.

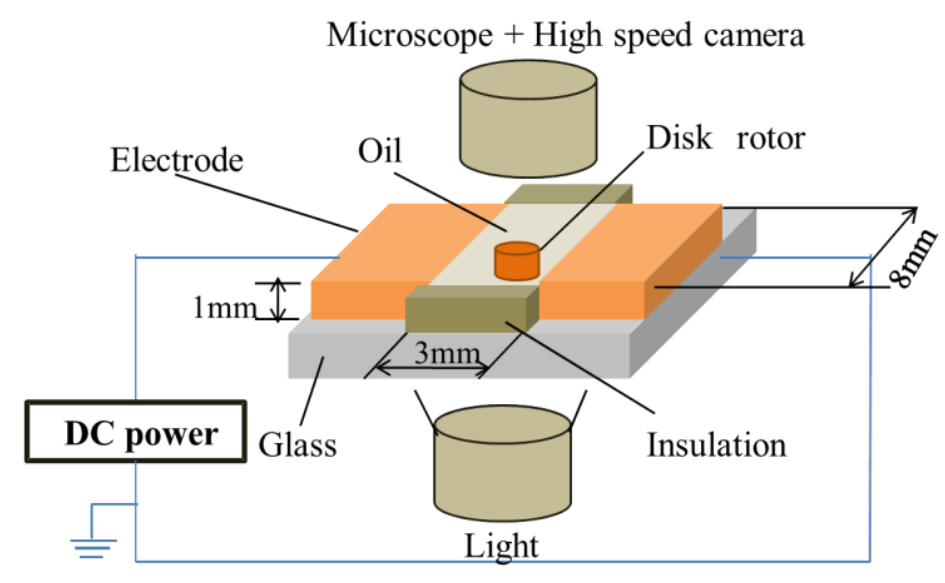

Figure 2: Experimental set up to determine the dependence of rotational speed on the DC field intensity

\section{Results and Discussion}

We have studied the angular motion of the epoxy rotors and have concluded that their dynamics is very complex. If the strength of static DC field is accounted for, three regimes have been observed.

1. Far below a threshold value $E_{c r}$ of the electric field, the disk does not show any motion.

2. Close to the threshold value and slightly above, the disk begin to rotate, but the angular displacement is less than $2 \pi$.

3. At DC field intensities higher than the threshold value, continuous rotation is observed. The direction of rotation seems to be randomly selected occurring either in clockwise or counter-clockwise direction.

\subsection{Electrorotation of polymer disk}

We first determined the dependence of spinning rate on the electric field intensity for the disk-shaped rotors. On Figure 3, it can be seen that with increasing electric field intensity, the rate of spinning significantly 
increases and within the experimental accuracy the dependence is linear. It is also seen that the threshold value $E_{c r}$ of the static electric field is close to $0.6 \mathrm{kV} / \mathrm{mm}$. Figure 3 also indicates that there is a significant size effect on the speed of rotation. At the same electric field intensity, the smallest disk (100 micron) performs the most intensive rotary motion. The opposite effect was found for the disk thickness. At the same electric field intensity, the rotation speed of the thicker disk (38 micron) is higher. The highest rotation speed that we were able to monitor was around $3000 \mathrm{rpm}$ which significantly exceeds the rotation speed of first electrostatic driven motor [1].

a

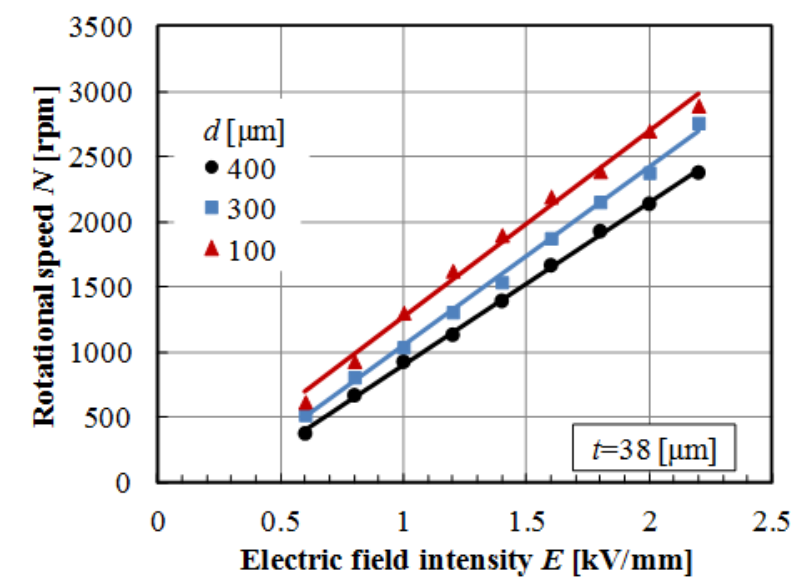

b

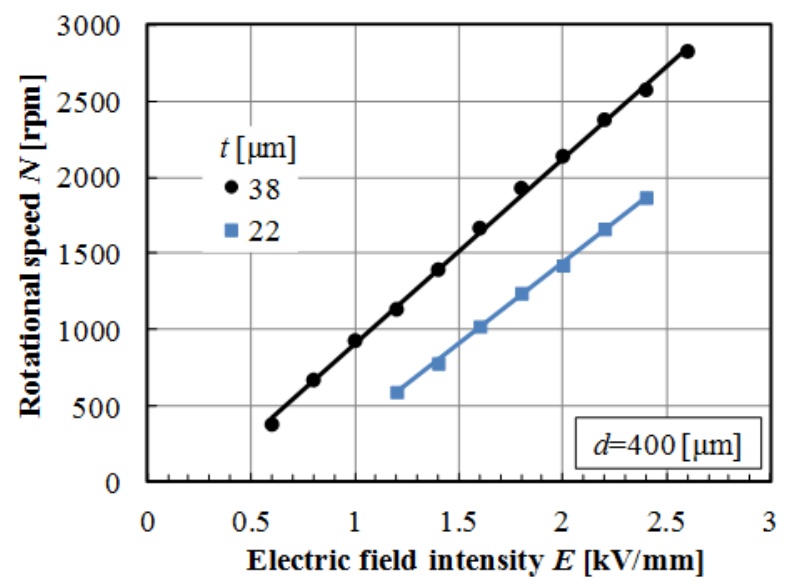

Figure 3: Dependence of rotational speed $N$ of polymer disks as a function of electric field intensity $E$. (a) Effect of disk diameter $d$ at constant thickness $(t=38 \mu \mathrm{m})$, (b) Effect of disk thickness $t$ at constant diameter $(d=400 \mu \mathrm{m})$. The solid line is guide for eyes. The diameter $d$ of the disks is indicated in the Figure.

\subsection{Electrorotation of hollow cylinder}

To study the effect of shape on the rotation phenomena, hollow cylinders were also prepared and put into uniform electric field. The inner, $d_{2}$ and outer, $d_{1}$ diameters were varied in a constant hollow rate of $16 \%$. Figure 4 shows how the size of hollow cylinder influences the speed of rotation. The thickness of both hollow cylinders was the same $(38 \mu \mathrm{m})$. It is seen on the graph that at the same electric field intensity, the smaller hollow cylinder $(400 / 160 \mu \mathrm{m})$ shows more intensive rotary motion similarly to the rotation of polymer disks. The random orientation of rotation was also observed. 


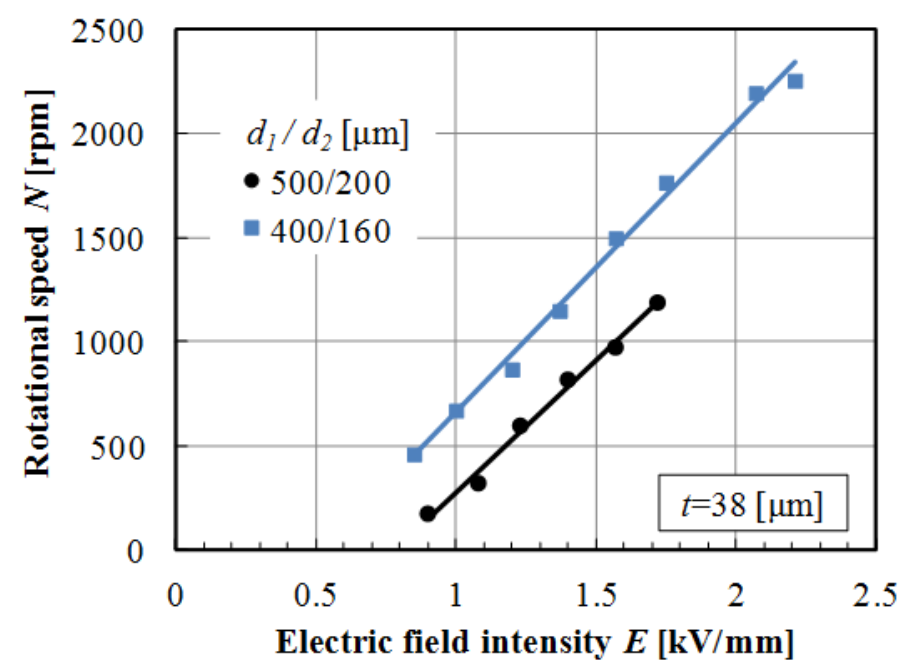

Figure 4: Dependence of rotational speed $N$ of hollow cylinders as a function of electric field intensity $E$ shape on the shape. The outer $d_{1}$ and inner $d_{2}$ diameters given in $\mu m$ are indicated in the Figure. The thickness of the hollow cylinders is $t=38 \mu \mathrm{m}$

\subsection{Electrorotation of polymer gear.}

In the previous examples, we always observed random selection of direction of rotation for disks and hollow cylinders. For any technical application, it is important to control the direction. In order to generate controlled rotation direction, we have to break the symmetry of the rotors. This can be achieved by using gears with asymmetric teeth. The asymmetric teeth were shaped using different angles on the drive side and coast side of the teeth (see Figure 1(c)). Due to the presence of asymmetric rotor teeth, we always observed directed rotation for these rotors. Figure 5 also indicates that there is a significant size effect on the speed of rotation. At the same electric field intensity, the smaller gear (300 micron) performs more intensive rotary motion. It is also seen that the linear dependence of the rate on the electric field intensity also remained.

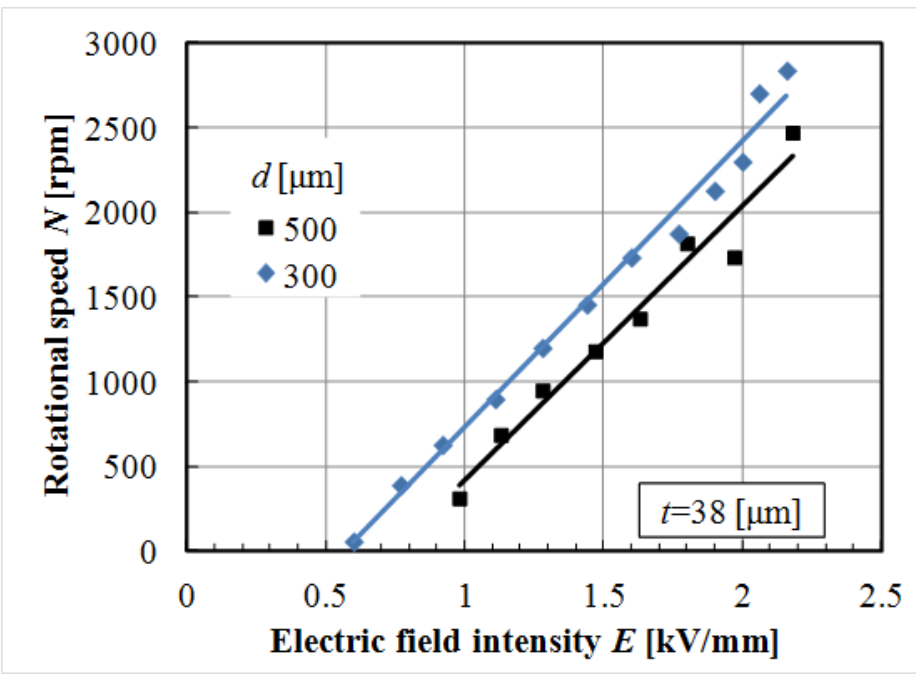

Figure 5: Dependence of rotational speed $N$ of polymer gear wheels as a function of electric field intensity $E$ on the diameter $d$. The thickness of the gears is $t=38 \mu \mathrm{m}$ 
As seen in Figure 6, when comparing the speed of rotation of polymer disks with gears with asymmetric teeth, it is worth to mention that the presence of teeth does not significantly decrease the intensity of rotation, even though with decreasing the electric field intensity the rotational speed of the gear very slightly decreases compared with that of the disk.

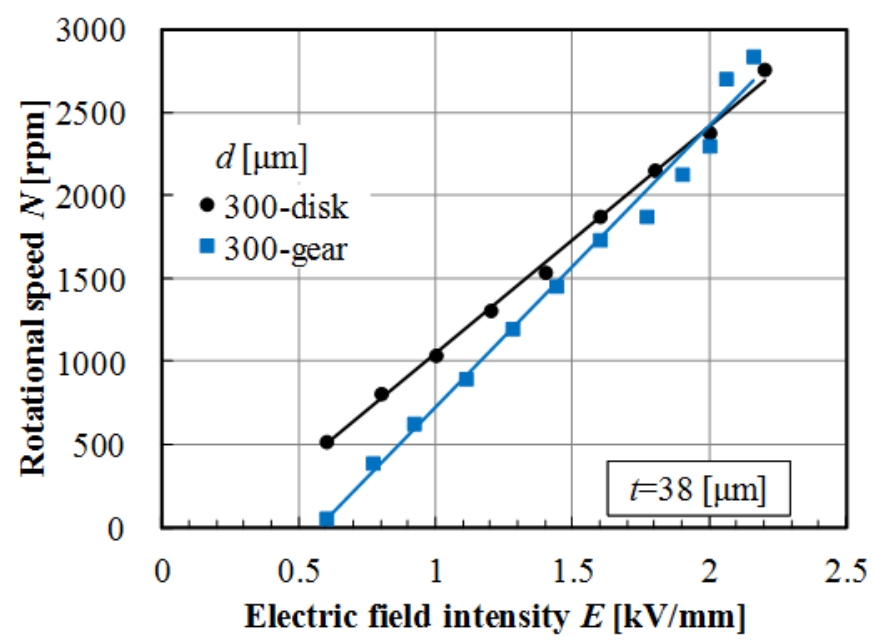

Figure 6: Comparison of rotational speed $N$ as a function of electric field intensity $E$ between polymer disk and polymer gear wheel of the same diameter $(d=300 \mu \mathrm{m})$ and thickness $(t=38 \mu \mathrm{m})$

\subsection{How small can we go?}

The first impulse to build micro- or nanomotor was given by R.P. Feynman in 1959 [13]. Since then MEMS field has grown enormously in the past decades. Despite the rapid development, very little was done at sizes below ten micrometer. It is a challenging task to build device and study the rotary behavior. In our work the diameter of the smallest rotor was $100 \mu \mathrm{m}$ with a thickness of $22 \mu \mathrm{m}$. This is far above the nano-sized range. Despite, this work clearly indicates that decreasing the size of the rotor, the speed of rotation increases as shown in Figure 7. This is holding a great promise to construct motors of even several micrometers diameter that can rotate even faster than presented here. Unfortunately no theoretical prediction is available which could help the design of micro- and nano-rotors. 


\section{Concluding remarks}

We have presented a new concept for the controlled rotation of polymer rotors with sizes ranging from centimeters to tenths of micrometers. The first direct observation of DC electric field induced rotation of epoxy based electrostatic micro rotors is presented.

Disk, hollow cylinders and gear with asymmetric teeth of different size as prototypes were successfully manufactured by mask lithography technique. The sub-millimeter sized rotors perform very intensive rotation in uniform DC electric field. It was observed that above a critical value of electric field intensity, the rotors begin to rotate at constant rate. This rate is sensitive to the field intensity. With increasing field intensities the angular velocity of rotating disk increases. This increase can be approximated by linear dependence. The size and shape of the rotors as well as the strength of electric field intensity determines the electro-mechanical performance of the rotors. The extrapolation of the observed trends suggests that motors of several micrometers diameter could be built that could rotate with at least the rate presented here. Regarding the driving principle of the micromotors presented here, it may open new perspectives for their use in several MEMS applications.

\section{Acknowledgements}

This research was partially supported by the JSPS Grant-in-Aid for Challenging Exploratory Research (Grant No. 26630084, 2014-2015), the Collaborative Research Project of Institute of Fluid Science, Tohoku University and the OTKA Foundation (Grant No. NK 101704 and NN 102624).

\section{References}

[1] Fan L S, Tai Y C and Muller R C 1988 Proc. IEEE International Electron Devices Meeting (San Francisco, USA) pp 666-669

[2] Ghalichechian N, Modafe A, Beyaz M I and Ghodssi R 2008 J. Microelectromech. Syst. 17 pp 632-642 
[3] Vinhais H F, de Godoy P H, Silva E C N 2006 ABCM Symposium Series in Mechatronics (Ouro Preto, Brasil) vol 2 pp 433-450

[4] Nicolaescu M A and Campeanu A $20076^{\text {th }}$ International Conference on Electromechanicaland Power Systems (Chisinau, Rep. Moldova) pp 421-424

[5] Loget G and Kuhn A 2011 Nat. Commun. 2:535 doi: 10.1038/ncomms 1550

[6] Quincke G 1896 Ann. Phys. Chem.59 pp 417-486

[7] Jones T B 1995 Electromechanics of Particles (Cambridge: Cambridge University Press)

[8] Turcu I J 1987 Phys. A: Math. Gen. 20 pp 3301-3307

[9] Peters F, Lobry L, Khayari A and Lemaire E 2009 J. Chem. Phys. 130 p 194905

[10] Antal M, Filipcsei G and Zrínyi M 2007 Compos. Sci. Technol. 67 pp 2884-2885

[11] Zrínyi M, Nakano M and Tsujita T 2012 Smart. Mater. Struct. 21 pp 1-6

[12] Zrínyi M 2011 Colloids Surf., A 382 pp 192-197

[13] Feynman R 1993 J. Microelectromech. Syst. 2 pp 4-14 